\title{
A Favard theorem for orthogonal rational functions on the unit circle
}

\author{
Adhemar Bultheel * \\ Pablo González-Vera ${ }^{\dagger}$ \\ Erik Hendriksen $\ddagger$ \\ Olav Njåstad $\S$
}

March 21, 2000

\begin{abstract}
We consider for $n=0,1, \ldots$ the nested spaces $\mathcal{L}_{n}$ of rational functions of degree $n$ at most with given poles $1 / \bar{\alpha}_{i},\left|\alpha_{i}\right|<1, i=1, \ldots, n$. Given a finite measure supported on the unit circle, we associate with it a nested orthogonal basis of rational functions $\phi_{0}, \ldots, \phi_{n}$ for $\mathcal{L}_{n}$, $n=0,1, \ldots$ These $\phi_{n}$ satisfy a recurrence relation that generalizes the recurrence for Szegö polynomials.

In this paper we shall prove a Favard type theorem which says that if you have a sequence of rational functions $\phi_{n} \in \mathcal{L}_{n}$ which are generated by such a recurrence, then there will be a measure $\mu$ supported on the unit circle to which they are orthogonal. We shall give a sufficient condition for the uniqueness of this measure.
\end{abstract}

\section{Introduction}

We consider for $n=0,1, \ldots$ the nested spaces $\mathcal{L}_{n}$ of rational functions of degree $n$ at most which are spanned by the basis of partial Blaschke products $\left\{B_{k}\right\}_{k=0}^{n}$ where $B_{0}=1, B_{n}=B_{n-1} \zeta_{n}$ for $n=1,2, \ldots$ and the Blaschke factors are defined as

$$
\zeta_{n}(z)=\frac{\bar{\alpha}_{n}}{\left|\alpha_{n}\right|} \frac{\alpha_{n}-z}{1-\bar{\alpha}_{n} z} \text { for }\left|\alpha_{n}\right|<1 .
$$

By convention we set $\bar{\alpha}_{n} /\left|\alpha_{n}\right|=-1$ for $\alpha_{n}=0$.

These spaces of rationals have been studied for example in connection with the Pick-Nevanlinna problem $[17,18,19,13,14,15,16]$ and in many applications $[2,20,3,7,4,5,6,8]$. Note that if all the $\alpha_{i}$ are equal to zero, the spaces $\mathcal{L}_{n}$ collapse to the spaces of polynomials of degree $n$.

Next we consider a finite positive measure $\mu$ on the unit circle and orthogonalize the basis with respect to the inner product

$$
\langle f, g\rangle_{\mu}=\int_{-\pi}^{\pi} f\left(e^{\mathbf{i} \theta}\right) \overline{g\left(e^{\mathbf{i} \theta}\right)} d \mu(\theta)
$$

to generate an orthonormal system $\phi_{0} \in \mathcal{L}_{0}$ and $\phi_{n} \in \mathcal{L}_{n} \backslash \mathcal{L}_{n-1}, n=1,2, \ldots$ It has been proved $[3,7,4,5,6]$ that these $\phi_{i}$ satisfy a recurrence relation that generalizes the recurrence for Szegö polynomials.

\footnotetext{
${ }^{*}$ Department of Computer Science, K.U.Leuven, Belgium

${ }^{\dagger}$ Department of Mathematical Analysis, University of La Laguna, Tenerife, Spain

${ }^{\ddagger}$ Department of Mathematics, University of Amsterdam, The Netherlands

${ }^{\S}$ Department of Mathematics, University of Trondheim-NTH, Norway
} 
In this paper we shall prove a Favard type theorem which says that if you have a sequence of rational functions $\phi_{n} \in \mathcal{L}_{n}$ which are generated by such a recurrence, then there will be a measure $\mu$ supported on the unit circle to which they are orthogonal.

The present paper completes in several respects earlier papers $[11,5]$ where such a theorem had already been given.

Most important is that in the earlier papers, there were only a finite number of different points $\alpha_{k}$ that were cyclically repeated. Here we shall discard this condition and let the points vary freely inside the unit disk.

A formal difference is that the orthogonality considered in $[11,5]$ is with respect to a linear functional (the moment functional) whereas here we shall work with an inner product defined by a measure supported on the unit circle. But it is again new that we shall give in this paper a sufficient condition for the uniqueness of this measure. In the previous papers, uniqueness has not been considered, but in the repetitive case, this condition will always be satisfied.

Another difference between the papers is the choice of the recurrence relations. In [11], the recurrence relations used are rational generalizations of the three-term recurrence relations of orthogonal polynomials while in [5] and also in the present paper, they are generalizations of the Szegö recurrence for polynomials orthogonal on the unit circle.

In $[11,5]$, the moment functional was found by direct construction. Also here we shall use a constructive approach for the measure which follows closely the elegant proof for the Szegö polynomials which was recently given in [9].

In the polynomial case, uniqueness of the measure follows easily because the polynomials are dense in the set of continuous functions. To obtain density of the rationals, one needs an extra condition on the numbers $\alpha_{k}$.

\section{The recurrence relations}

We shall need the following notations and assumptions. $\mathbf{D} \subset \mathbf{C}$ is used to denote the open unit disc of complex numbers and $\mathbf{T}$ denotes its boundary, the unit circle. Let $\mu$ be a finite positive measure on $\mathbf{T}$ whose support is an infinite set. Hence the distribution function $\mu(t)=\int_{0}^{t} d \mu$ has an infinite number of points of increase. Suppose it is normalized so that $\int_{\mathbf{T}} d \mu=1$. The normalized Lebesgue measure for the unit circle is denoted by $\lambda: d \lambda(\theta)=(2 \pi)^{-1} d \theta$. For any measure $\nu$ on $\mathbf{T}$, the inner product $\langle\cdot, \cdot\rangle_{\nu}$ is defined by

$$
\langle f, g\rangle_{\nu}=\int_{-\pi}^{\pi} f\left(e^{\mathbf{i} \theta}\right) \overline{g\left(e^{\mathbf{i} \theta}\right)} d \nu(\theta)
$$

If $\nu$ is replaced by $\lambda$, we drop the subscript. The metric space $L_{2}(\mu)$ of square integrable functions is well known. When $\mu$ is replaced by $\lambda$, we simply denote it as $L_{2}$. The Hardy subspace of functions analytic in $\mathbf{D}$ is denoted by $H_{2}$. The Nevanlina class $N$ is the set of ratios $g / h$ with $g, h \in H_{\infty}$ it contains all Hardy spaces $H_{p}, 0<p \leq \infty$.

$\mathcal{B}$ is the unit ball of holomorphic functions in the unit disc:

$$
\mathcal{B}=\{f \text { holomorphic in } \mathbf{D} \text { and }|f|<1\} .
$$

$P(z, w)$ denotes the Poisson kernel

$$
P(z, w)=\frac{1-|w|^{2}}{|z-w|^{2}}
$$

We also introduce the substar transformation as $f_{*}(z)=\overline{f(1 / \bar{z})}$. 
For $\alpha_{i} \in \mathbf{D}, \zeta_{i}(z)$ denotes the Blaschke factor (1.1) and $B_{0}=1, B_{n}=B_{n-1} \zeta_{n}$ are finite Blaschke products. Recall $\bar{\alpha}_{i} /\left|\alpha_{i}\right|=-1$ if $\alpha_{i}=0$. We shall also use the notation $B_{n \backslash m}$ to mean $B_{n} / B_{m}$ where $m \leq n . \mathcal{L}_{n}=\operatorname{span}\left\{B_{k}: k=0, \ldots, n\right\}$ is the space of rational functions with poles $1 / \bar{\alpha}_{i}$.

If $f \in \mathcal{L}_{n}$, then we define $f^{*}(z)=B_{n}(z) f_{*}(z) \in \mathcal{L}_{n}$.

By $\phi_{n}$ we shall denote rational functions $\phi_{0} \in \mathcal{L}_{0}$ and $\phi_{n} \in \mathcal{L}_{n} \backslash \mathcal{L}_{n-1}, n \geq 1$ which are orthonormal with respect to $\langle\cdot, \cdot\rangle_{\mu}$. We shall suppose that their leading coefficients $\kappa_{n}$ with respect to the basis $B_{0}, \ldots, B_{n}$ are positive, namely $\kappa_{n}=\overline{\phi_{n}^{*}\left(\alpha_{n}\right)}>0$, by which the $\phi_{n}$ are now uniquely defined.

$J$ denotes a $2 \times 2$ indefinite signature matrix $J=1 \oplus-1$. A matrix $\theta=\left[\theta_{i j}\right] \in N^{2 \times 2}$ is called $J$-unitary if $\theta_{*} J \theta=J$ a.e. where the substar for a matrix is defined by

$$
\left[\begin{array}{cc}
\theta_{11} & \theta_{12} \\
\theta_{21} & \theta_{22}
\end{array}\right]_{*}=\left[\begin{array}{cc}
\theta_{11 *} & \theta_{21 *} \\
\theta_{12 *} & \theta_{22 *}
\end{array}\right]
$$

Such a matrix $\theta$ is called $J$-contractive (in $\mathbf{D}$ ) if $\theta^{H} J \theta \leq J$ a.e. in $\mathbf{D}$, where the superscript ${ }^{H}$ denotes complex conjugate transpose. Note that for $z \in \mathbf{T}, \theta_{*}(z)=[\theta(z)]^{H}$.

A result from Dewilde and Dym [7, p.448] says that if $\theta$ is $J$-contractive, then

$$
\left(\theta_{22}+\theta_{21}\right)^{-1} \in H_{2}
$$

The following type of recurrence relation has been proved in $[4,6]$ (the expression for $e_{n}^{2}$ is only implicit in [4])

Theorem 2.1 For the orthonormal basis functions in $\mathcal{L}_{n}$, a recursion of the following form exists

$$
\left[\begin{array}{l}
\phi_{n}(z) \\
\phi_{n}^{*}(z)
\end{array}\right]=N_{n} \frac{1-\bar{\alpha}_{n-1} z}{1-\bar{\alpha}_{n} z}\left[\begin{array}{cc}
1 & \bar{\lambda}_{n} \\
\lambda_{n} & 1
\end{array}\right]\left[\begin{array}{cc}
\zeta_{n-1}(z) & 0 \\
0 & 1
\end{array}\right]\left[\begin{array}{l}
\phi_{n-1}(z) \\
\phi_{n-1}^{*}(z)
\end{array}\right]
$$

where the matrix $N_{n}$ is a constant $e_{n}>0$ times a unitary matrix

$$
N_{n}=e_{n}\left[\begin{array}{cc}
\eta_{n}^{1} & 0 \\
0 & \eta_{n}^{2}
\end{array}\right]
$$

with $\eta_{n}^{1}$ and $\eta_{n}^{2} \in \mathbf{T}$. The constant $\eta_{n}^{1}$ is chosen such that $\phi_{n}^{*}\left(\alpha_{n}\right)>0$. The other constant $\eta_{n}^{2}$ is related to $\eta_{n}^{1}$ by

$$
\eta_{n}^{2}=\overline{\eta_{n}^{1}} \frac{\alpha_{n-1}}{\left|\alpha_{n-1}\right|} \frac{\bar{\alpha}_{n}}{\left|\alpha_{n}\right|}
$$

The parameter $\lambda_{n}$ is given by

$$
\lambda_{n}=\eta \frac{\overline{\phi_{n}\left(\alpha_{n-1}\right)}}{\phi_{n}^{*}\left(\alpha_{n-1}\right)} \text { with } \eta=\frac{1-\alpha_{n} \bar{\alpha}_{n-1}}{1-\bar{\alpha}_{n} \alpha_{n-1}} \frac{\bar{\alpha}_{n}}{\left|\alpha_{n}\right|} \frac{\alpha_{n-1}}{\left|\alpha_{n-1}\right|} \in \mathbf{T}
$$

which can also be written as

$$
\lambda_{n}=\frac{\alpha_{n-1}}{\left|\alpha_{n-1}\right|} \frac{\left\langle\phi_{n-1}, \frac{z-\alpha_{n-1}}{1-\bar{\alpha}_{n} z} \phi_{n-1}\right\rangle_{\mu}}{\left\langle\phi_{n-1}, \frac{1-\bar{\alpha}_{n-1} z}{1-\bar{\alpha}_{n} z} \phi_{n-1}^{*}\right\rangle_{\mu}},
$$

and the value of $e_{n}>0$ can be obtained as the positive square root of

$$
e_{n}^{2}=\frac{1-\left|\alpha_{n}\right|^{2}}{1-\left|\alpha_{n-1}\right|^{2}} \frac{1}{1-\left|\lambda_{n}\right|^{2}}
$$

The formula (2.2) holds from $n=1$ on if we define $\alpha_{0}=0$, i.e. $\zeta_{0}(z)=z$. 
It will be useful to write an inverse form of the recursion formulas as in the next theorem which was also proved in $[4,6]$.

Theorem 2.2 Given the orthonormal function $\phi_{n}$ with $\phi_{n}^{*}\left(\alpha_{n}\right)=\kappa_{n}>0$, all the previous orthonormal functions $\phi_{k}, k<n$ are uniquely defined if they are normalized by $\phi_{k}^{*}\left(\alpha_{k}\right)=\kappa_{k}>0$. They can be found with the recursions

$$
\left[\begin{array}{c}
\phi_{n-1}(z) \\
\phi_{n-1}^{*}(z)
\end{array}\right]=V_{n}(z)\left[\begin{array}{c}
\phi_{n}(z) \\
\phi_{n}^{*}(z)
\end{array}\right]
$$

with

$$
V_{n}(z)=\frac{1}{1-\left|\lambda_{n}\right|^{2}} \frac{1-\bar{\alpha}_{n} z}{1-\bar{\alpha}_{n-1} z}\left[\begin{array}{cc}
1 / \zeta_{n-1}(z) & 0 \\
0 & 1
\end{array}\right]\left[\begin{array}{cc}
1 & -\bar{\lambda}_{n} \\
-\lambda_{n} & 1
\end{array}\right] N_{n}^{-1}
$$

with all the quantities appearing in this formula as in Theorem 2.1.

\section{Favard theorem for orthogonal functions}

We have seen how the orthogonal functions satisfied certain recurrence relations which generalize the Szegö recurrence relations. The converse is known as a Favard Theorem, named after Favard's paper [10]. Such a Favard Theorem states that if functions satisfy such recurrence relations, then they will be orthogonal rational functions with respect to a positive measure on the unit circle. A simple proof for the Szegö polynomials was recently given in [9]. There, not only the existence of a measure is proved, but it is shown to be unique and it is actually constructed during the proof. We shall follow in this section a similar approach for the rational case.

We shall give a sequence of lemmas which will eventually lead to the proof of the Favard Theorem. We start with the orthogonal functions.

Lemma 3.1 Suppose we are given two sequences of numbers $\alpha_{k} \in \mathbf{D}$ and $\lambda_{k} \in \mathbf{D}$ for $k=1,2, \ldots$ and set $\alpha_{0}=0$. Define the numbers $e_{k}>0$ by their squares

$$
e_{k}^{2}=\frac{1-\left|\alpha_{k}\right|^{2}}{1-\left|\alpha_{k-1}\right|^{2}} \frac{1}{1-\left|\lambda_{k}\right|^{2}} \text { for } k=1,2, \ldots
$$

Finally define the functions $\phi_{k}$ by

$$
\phi_{0}=1, \quad \phi_{k}=e_{k} \eta_{k}^{1} \frac{1-\bar{\alpha}_{k-1} z}{1-\bar{\alpha}_{k} z}\left[\zeta_{k-1} \phi_{k-1}+\bar{\lambda}_{k} \phi_{k-1}^{*}\right], \quad k=1,2, \ldots
$$

where the numbers $\eta_{k}^{1} \in \mathbf{T}$ are chosen such that $\phi_{k}^{*}\left(\alpha_{k}\right)>0$.

Then the functions $\phi_{k}^{*}$ satisfy the following recurrence

$$
\phi_{0}^{*}=1, \quad \phi_{k}^{*}=e_{k} \eta_{k}^{2} \frac{1-\bar{\alpha}_{k-1} z}{1-\bar{\alpha}_{k} z}\left[\zeta_{k-1} \lambda_{k} \phi_{k-1}+\phi_{k-1}^{*}\right], \quad k=1,2, \ldots
$$

with

$$
\eta_{k}^{2}=\bar{\eta}_{k}^{1} \frac{\alpha_{k-1}}{\left|\alpha_{k-1}\right|} \frac{\bar{\alpha}_{k}}{\left|\alpha_{k}\right|}
$$

Moreover, $1 / \phi_{n}^{*} \in H_{2}$.

Proof. We can leave the first part of the proof to the reader because it comes down to simple calculus. For the second part note that we can couple the two recurrences (3.2) and (3.3) into the form (2.2). We can use this form recursively and end up with (recall $\alpha_{0}=0$ )

$$
\left[\begin{array}{l}
\phi_{n} \\
\phi_{n}^{*}
\end{array}\right]=\frac{c_{n}}{1-\bar{\alpha}_{n} z} \Theta_{n}\left[\begin{array}{l}
1 \\
1
\end{array}\right]
$$


where $c_{n}=\prod_{k=1}^{n} e_{k}$ and where $\Theta_{n}$ is given by $\Theta_{n}=\theta_{n} \theta_{n-1} \cdots \theta_{1}$ with

$$
\theta_{k}=\left[\begin{array}{cc}
\eta_{k}^{1} & 0 \\
0 & \eta_{k}^{2}
\end{array}\right]\left[\begin{array}{cc}
1 & \bar{\lambda}_{k} \\
\lambda_{k} & 1
\end{array}\right]\left[\begin{array}{cc}
\zeta_{k-1}(z) & 0 \\
0 & 1
\end{array}\right]
$$

which is $J$-contractive being the product of $J$-contractive factors. Hence, because

$$
\phi_{n}^{*}=\frac{c_{n}}{1-\bar{\alpha}_{n} z}\left[\left(\Theta_{n}\right)_{21}+\left(\Theta_{n}\right)_{22}\right]
$$

it follows by property (2.1) that

$$
1 / \phi_{n}^{*}=c_{n}^{-1}\left(1-\bar{\alpha}_{n} z\right)\left[\left(\Theta_{n}\right)_{21}+\left(\Theta_{n}\right)_{22}\right]^{-1} \in H_{2}
$$

which concludes the proof.

Lemma 3.2 Under the conditions of Lemma 3.1, it holds that

$$
\lambda_{k}=\eta \frac{\overline{\phi_{k}\left(\alpha_{k-1}\right)}}{\phi_{k}^{*}\left(\alpha_{k-1}\right)} \text { with } \eta=\frac{\bar{\alpha}_{k}}{\left|\alpha_{k}\right|} \frac{\alpha_{k-1}}{\left|\alpha_{k-1}\right|} \frac{1-\alpha_{k} \bar{\alpha}_{k-1}}{1-\bar{\alpha}_{k} \alpha_{k-1}} \in \mathbf{T} .
$$

Proof. From the recurrences for $\phi_{k}$ and $\phi_{k}^{*}$, we find

$$
\overline{\phi_{k}\left(\alpha_{k-1}\right)}=e_{k} \bar{\eta}_{k}^{1} \frac{1-\left|\alpha_{k-1}\right|^{2}}{1-\alpha_{k} \bar{\alpha}_{k-1}}\left[0+\lambda_{k} \phi_{k-1}^{*}\left(\alpha_{k-1}\right)\right]
$$

and

$$
\phi_{k}^{*}\left(\alpha_{k-1}\right)=e_{k} \eta_{k}^{2} \frac{1-\left|\alpha_{k-1}\right|^{2}}{1-\bar{\alpha}_{k} \alpha_{k-1}}\left[0+\phi_{k-1}^{*}\left(\alpha_{k-1}\right)\right]
$$

Dividing (3.6) by (3.7) gives

$$
\frac{\overline{\phi_{k}\left(\alpha_{k-1}\right)}}{\phi_{k}^{*}\left(\alpha_{k-1}\right)}=\frac{\bar{\eta}_{k}^{1}}{\eta_{k}^{2}} \frac{1-\bar{\alpha}_{k} \alpha_{k-1}}{1-\alpha_{k} \bar{\alpha}_{k-1}} \lambda_{k}=\frac{\bar{\alpha}_{k-1}}{\left|\alpha_{k-1}\right|} \frac{\alpha_{k}}{\left|\alpha_{k}\right|} \frac{1-\bar{\alpha}_{k} \alpha_{k-1}}{1-\alpha_{k} \bar{\alpha}_{k-1}} \lambda_{k}=\bar{\eta} \lambda_{k} .
$$

The result now follows.

Lemma 3.3 Define the positive measure $\mu_{n}$ on $\mathbf{T}$ by

$$
d \mu_{n}(z)=\frac{P\left(z, \alpha_{n}\right)}{\left|\phi_{n}(z)\right|^{2}} d \lambda(z)
$$

with $P(z, w)$ the Poisson kernel. Then the functions constructed in Lemma 3.1 satisfy the orthogonality relations

$$
\left\langle\phi_{k}, \phi_{\ell}\right\rangle_{\mu_{n}}=\delta_{k \ell} \text { for } 0 \leq k, \ell \leq n .
$$

Proof. We shall first prove that $\phi_{n}$ is orthonormal with respect to all its predecessors :

$$
\left\langle\phi_{n}, \phi_{m}\right\rangle_{\mu_{n}}=\delta_{n m} \text { for } 0 \leq m \leq n \text {. }
$$

This is shown as follows

$$
\begin{aligned}
\left\langle\phi_{n}, \phi_{m}\right\rangle_{\mu_{n}} & =\int \frac{\phi_{n}(z) \phi_{m *}(z)}{\phi_{n}(z) \phi_{n *}(z)} P\left(z, \alpha_{n}\right) d \lambda(z) \\
& =\int \frac{\phi_{m *}(z)}{\phi_{n *}(z)} P\left(z, \alpha_{n}\right) d \lambda(z) \\
& =\int B_{n \backslash m}(z) \frac{\phi_{m}^{*}(z)}{\phi_{n}^{*}(z)} P\left(z, \alpha_{n}\right) d \lambda(z) \\
& =\left.B_{n \backslash m}(z) \frac{\phi_{m}^{*}(z)}{\phi_{n}^{*}(z)}\right|_{z=\alpha_{n}}=\delta_{n m} .
\end{aligned}
$$


The general orthogonality follows from Theorem 2.2 which says that all the previous orthogonal functions are defined in terms of $\phi_{n}$, orthonormal to $\mathcal{L}_{n-1}$, by the inverse recurrence, and we have just proved by the previous Lemma 3.2 that the recurrence in Lemma 3.1 is the same as the one from Theorem 2.1.

One final result we shall need is

Lemma 3.4 Let us define $\mathcal{R}_{n}=\mathcal{L}_{n}+\mathcal{L}_{n *}$ where $\mathcal{L}_{n *}=\left\{f_{*}: f \in \mathcal{L}_{n}\right\}$, and $\mathcal{R}=\mathcal{R}_{\infty}$, then $\mathcal{R}$ is dense in the set $C(\mathbf{T})$ of continuous functions on $\mathbf{T}$ iff $\sum\left(1-\left|\alpha_{k}\right|\right)=\infty$.

Proof. This is a direct consequence of the "closure criterion" discussed in Addendum A.2 of [1, p. 244].

We are now ready to prove the following Favard-type Theorem.

Theorem 3.5 There exists a Borel measure on $\mathbf{T}$ for which the $\phi_{n}$ as constructed in Lemma 3.1 are the orthonormal functions. If the Blaschke condition $\sum\left(1-\left|\alpha_{k}\right|\right)=\infty$ is satisfied, the measure is unique.

Proof. Define

$$
\mu_{n}(t)=\int_{0}^{t} \frac{P\left(e^{\mathbf{i} \theta}, \alpha_{n}\right)}{\left|\phi_{n}\left(e^{\mathbf{i} \theta}\right)\right|^{2}} d \lambda(\theta)=\int_{0}^{t} d \mu_{n}(\theta) .
$$

These are all increasing functions and uniformly bounded $\left(\int d \mu_{n}=1\right)$. Hence, there exists a subsequence such that

$$
\lim _{k \rightarrow \infty} \mu_{n_{k}}(\theta)=\mu(\theta) \text { and } \lim _{k \rightarrow \infty} \int f\left(e^{\mathbf{i} \theta}\right) d \mu_{n_{k}}(\theta)=\int f d \mu
$$

for all functions $f$ continuous on $\mathbf{T}$. Thus the $\left\{\phi_{n}\right\}$ are an orthonormal system with respect to this measure $\mu$. Since the linear functional defined on $\mathcal{R}$ through

$$
\Phi\left(\phi_{k} \phi_{l *}\right)=\delta_{k l}, \quad k, l=0,1, \ldots
$$

exists and is bounded. The uniqueness now follows from Lemma 3.4 and the representation of bounded linear functionals on $C(\mathbf{T})$ (F. Riesz).

Note: The Blaschke condition $\sum\left(1-\left|\alpha_{k}\right|\right)=\infty$ is equivalent to the rationals $\mathcal{R}$ being dense in the set $C(\mathbf{T})$. However, the latter is not necessary for the uniqueness of the measure $\mu$. Note that when there is only a finite number of different points $\alpha_{k}$ some of which are repeated an infinite number of times, the Blaschke condition is satisfied and uniqueness of the measure holds.

The question of weaker, sufficient conditions ensuring uniqueness, is related to the interpolation problem being determinate and the convergence of multipoint Padé approximants. This is a much more difficult question which shall be treated in another paper. See in this respect [12] for the Stieltjes case.

\section{References}

[1] N.I. Achieser. Theory of approximation. Frederick Ungar Publ. Co., New York, 1956.

[2] N.I. Akhiezer. The classical moment problem. Oliver and Boyd, Edinburgh, 1969. Originally published Moscow, 1961.

[3] A. Bultheel and P. Dewilde. Orthogonal functions related to the Nevanlinna-Pick problem. In P. Dewilde, editor, Proc. 4th Int. Conf. on Math. Theory of Networks and Systems at Delft, pages 207-212, North-Hollywood, 1979. Western Periodicals. 
[4] A. Bultheel, P. González-Vera, E. Hendriksen, and O. Njåstad. A Szegő theory for rational functions. Technical Report TW131, Computer Science Department, K.U.Leuven, May 1990.

[5] A. Bultheel, P. González-Vera, E. Hendriksen, and O. Njåstad. Orthogonal rational functions similar to Szegő polynomials. In C. Brezinski, L. Gori, and A. Ronveaux, editors, Orthogonal polynomials and their applications, volume 9 of IMACS annals on computing and applied mathematics, pages 195-204, Basel, 1991. J.C. Baltzer AG.

[6] A. Bultheel, P. González-Vera, E. Hendriksen, and O. Njåstad. The computation of orthogonal rational functions and their interpolating properties. Numerical Algorithms, 2(1):85-118, 1992.

[7] P. Dewilde and H. Dym. Schur recursions, error formulas, and convergence of rational estimators for stationary stochastic sequences. IEEE Trans. on Information Theory, IT-27:446-461, 1981.

[8] M.M. Djrbashian. A survey on the theory of orthogonal systems and some open problems. In P. Nevai, editor, Orthogonal polynomials: Theory and practice, volume 294 of Series C: Mathematical and Physical Sciences, pages 135-146, Boston, 1990. NATO-ASI, Kluwer Academic Publishers.

[9] T. Erdélyi, P. Nevai, J. Zhang, and J.S. Geronimo. A simple proof of "Favard's theorem" on the unit circle. Atti. Sem. Mat. Fis. Univ. Modena, 29:41-46, 1991. Proceedings of the Meeting "Trends in Functional Analysis and Approximation Theory", 1989, Estratti Dagli Atti del Seminario Matematico e Fisico dell'Universitá di Modena, Italy.

[10] J. Favard. Sur les polynomes de Tchebicheff. Comptes Rendus de l'Académie des Sciences, Paris, 200:2052-2053, 1935.

[11] E. Hendriksen and O. Njåstad. A Favard theorem for rational functions. J. Math. Anal. Appl., 142(2):508-520, 1989.

[12] G.L. López. Conditions for convergence of multipoint Padé approximants for functions of Stieltjes type. Math. USSR Sbornik, 35:363-376, 1979.

[13] R. Nevanlinna. Über beschränkte Funktionen die in gegebenen Punkten vorgeschriebene werte annehmen. Ann. Acad. Sci. Fenn. Ser. A., 13(1):71pp., 1919.

[14] R. Nevanlinna. Asymptotische Entwickelungen beschränkter Funktionen und das Stieltjessche Momentenproblem. Ann. Acad. Sci. Fenn. Ser. A., 18(5):53pp., 1922.

[15] R. Nevanlinna. Kriterien für die Randwerte beschränkter Funktionen. Math. Z., 13:1-9, 1922.

[16] R. Nevanlinna. Über beschränkte analytische Funktionen. Ann. Acad. Sci. Fenn. Ser. A., 32(7):75pp., 1929.

[17] G. Pick. Über die Beschränkungen analytischen Funktionen Welche durch vorgegebene Funktionswerte bewirkt werden. Math. Ann., 77:7-23, 1916.

[18] G. Pick. Über die Beschränkungen analytischen Funktionen durch vorgegebene Funktionswerte. Math. Ann., 78:270-275, 1918.

[19] G. Pick. Über beschränkte Funktionen mit vorgeschriebenen Wertzuordnungen. Ann. Acad. Sci. Fenn. Ser. A, 15(3):17pp., 1920.

[20] J. L. Walsh. Interpolation and approximation, volume 20 of AMS Colloquium publications. American Mathematical Society, Providence, Rhode Island, 3rd edition, 1960. First edition 1935. 